\title{
A massagem modeladora na remodelagem da circunferência abdominal em mulheres: revisão sistemática
}

A massagem modeladora é atualmente utilizada em tratamentos estéticos e demonstra um resultado razoável durante as sessões realizadas na área da fisioterapia principalmente a dermatofuncional, quando utiliza as terapias manuais com o propósito de facilitar a remodelação da circunferência da cintura abdominal, pode proporcionar a melhora da aparência física e da saúde. Realizar uma revisão sistemática de literatura sobre a massagem modeladora na remodelagem da circunferência abdominal em mulheres. Os estudos foram selecionados a partir das seguintes bases de dados eletrônicos: PubMed, CAPES, ERIC, Embase e SciELO. A pesquisa foi realizada em novembro de 2019 com restrições de data da publicação dos últimos dez anos (2009-2019) e também com restrições quanto ao idioma: somente os estudos em português, inglês foram elegíveis. Foi realizada uma análise detalhada dos artigos, restando 7 estudos incluídos que preenchiam todos os critérios de inclusão de elegibilidade previamente estabelecidos, que obtiveram redução de medidas na região do abdômen. De acordo com os resultados obtidos neste estudo, pode-se afirmar de que a terapia manual da massagem modeladora é eficaz no tratamento da gordura localizada. Portanto, sugere-se a realização de novas pesquisas sobre essa temática e que seja de forma mais isolada, com maior número de amostras e maior tempo de tratamento para a validação do estudo.

Palavras-chave: Terapia Manual; Massagem Modeladora; Circunferência.

\section{Modeling massage in the reshaping of waist circumference in women: a systematic review}

\begin{abstract}
Modeling massage is currently used in aesthetic treatments and shows a reasonable result during sessions in the area of physiotherapy, especially dermatofunctional, when using manual therapies with the purpose of facilitating the remodeling of the waist circumference, it can provide the improvement of physical appearance and health. Conduct a systematic review of the literature on modeling massage in the reshaping of waist circumference in women. The studies were selected from the following electronic databases: PubMed, CAPES, ERIC, Embase and SciELO. The survey was carried out in November 2019 with restrictions on the date of publication of the last ten years (2009-2019) and also with restrictions on language: only studies in Portuguese, English were eligible. A detailed analysis of the articles was carried out, leaving 7 included studies that fulfilled all the eligibility inclusion criteria previously established, which obtained reduction of measures in the abdomen region. According to the results obtained in this study, it can be said that the manual therapy of the modeling massage is effective in the treatment of localized fat. Therefore, it is suggested to carry out further research on this topic and to do it in a more isolated way, with a larger number of samples and longer treatment time for the validation of the study.
\end{abstract}

Keywords: Manual therapy; Modeling Massage; Circumference.

Topic: Fisioterapia

Reviewed anonymously in the process of blind peer
Received: 06/06/2020

Approved: 17/08/2020
Renata Machado Penteado (iD

Universidade Regional Integrada do Alto Uruguai e das Missões, Brasil http://lattes.cnpq.br/2244961395288984

http://orcid.org/0000-0001-9581-9577

renatamp9621@gmail.com

Tânia Regina Warpechowski (iD

Universidade Regional Integrada do Alto Uruguai e das Missões, Brasil

http://lattes.cnpq.br/5042138395515098

http://orcid.org/0000-0003-0163-3339

twrfisio@yahoo.com.br
Referencing this:

PENTEADO, R. M.; WARPECHOWSKI, T. R.. A massagem modeladora na remodelagem da circunferência abdominal em mulheres: revisão sistemática. Scire Salutis, v.10, n.3, p.21-29, 2020. DOI: http://doi.org/10.6008/CBPC2236-9600.2020.003.0003 


\section{INTRODUÇÃO}

O índice de obesos cresceu no Brasil nos últimos anos, segundo levantamento realizado em 2018, houve um crescimento de $67,8 \%$ na população brasileira nos últimos treze anos. Foi identificado que as mulheres obtiveram um índice de crescimento maior que os homens, atingindo um aumento de $20,7 \%$ (BRASIL, 2018).

Segundo Pereira (2007), a gordura localizada na região abdominal, pode ser tanto a lipodistrofia quanto a hiperlipodistrofia, ocasiona a diminuição da autoestima e desconfortos físicos nas mulheres, mas acredita que tratamentos estéticos como a massagem modeladora podem contribuir para a remodelação da circunferência do abdômen, salientou que com o auxílio dos exercícios físicos o resultado é mais efetivo.

Gondim et al. (2018) reforçam que as terapias manuais são atualmente utilizadas em tratamentos estéticos e demonstram em seu trabalho um resultado razoável durante as sessões realizadas, perceberam algumas vantagens como na diminuição do estresse e tensões, aumento da nutrição tecidual e aceleração do metabolismo.

As células adiposas, metabolicamente ativas, se localizam especificamente nas regiões como o abdômen, flancos, quadris e pernas, havendo então o aumento de tamanho das células adiposas, ocasionando a compressão dos vasos sanguíneos, retenção de líquidos, além de diminuir o metabolismo, tudo isso repercute na dificuldade de diminuir medidas da circunferência abdominal (TRIFFONI et al., 2019).

Costa et al. (2017) acreditam que o fato da massagem modeladora exercer pressões e velocidades de maneira alternada, alcançando as camadas mais profundas da pele como os vasos sanguíneos, sistema linfático até aos vasos capilares, promove a dilatação e o aumento do fluxo na intraderme, favorecendo a melhora da vitalidade e da flexibilidade nos tecidos cutâneos, além de promover a diminuição da tensão muscular e auxilia na liberação de substâncias analgésicas.

Starkey (2001) reconheceu as técnicas da massagem modeladora como proporcionadora de ganhos ao corpo, por ter efeito calmante e por eliminar a tensão e ansiedade, pode reduzir os resíduos e dissolve os depósitos de gordura, podendo também suavizar as aderências e as cicatrizes. Quando aplicada sobre o abdômen, consegue ativar a circulação fecal, aumentando os movimentos peristálticos e acelerando o metabolismo, subsidiando o bem-estar para estes indivíduos.

De acordo com Cassar (2001), a eficácia da massagem modeladora é a ativação do rompimento das células adiposas e a estimulação da passagem destas células para o sistema linfático obtendo um resultado de redistribuição simétrica na área. Sendo assim, é um método atualmente utilizado na remodelação de medidas, que proporciona o aumento da circulação sanguínea e acelerando o metabolismo (FRANÇA et al., 2016).

Corroborando com isso, Tacani et al. (2010), afirmam que a massagem modeladora por ser uma técnica usada para gordura localizada, pois, consegue ultrapassar as camadas profundas, alcançando as células adiposas. No entanto, as manobras da massagem quando aplicadas, sobre os tecidos corporais promovem relaxamento, revigoramento local e sistêmico, por esta razão pode aumentar o fluxo sanguíneo 
e desfazer as aderências e melhorar o retorno venoso (STARKEY, 2001).

Machado et al. (2017) afirmam que:

A massagem modeladora promove benefícios como a oxigenação do tecido, quebra de cadeias de gordura e a melhora do tônus muscular, favorecendo assim não só a estética corporal, mas também agindo no psicológico do cliente diminuindo o estresse e proporcionando ao mesmo bem-estar com saúde.

Corroborando com esses trabalhos, acredita-se que a fisioterapia, principalmente a dermatofuncional, quando utiliza as terapias manuais com o propósito de facilitar a remodelação da circunferência da cintura abdominal, pode proporcionar a melhora da aparência física e da saúde dos indivíduos, podendo haver redução de medidas no abdômen e noutras regiões do corpo (MILANI et al., 2006; SILVA et al., 2011).

\section{METODOLOGIA}

Os estudos incluídos nesta revisão sistemática foram publicados em revistas nacionais e internacionais. Sendo incluídos nesta revisão, ensaios clínicos randomizados e não randomizados, estudos pilotos. Os critérios de inclusão foram estudos que avaliaram a efetividade da massagem modeladora na região abdominal em mulheres que possuíam gordura localizada. Os estudos excluídos foram os quais avaliaram outra parte do corpo ou que não utilizaram a técnica da massagem modeladora.

\section{Estratégia de pesquisa}

Para alcançar o objetivo desse estudo, foi realizada uma pesquisa bibliográfica nas seguintes bases eletrônicas: PubMed, CAPES, ERIC, LILASC, Embase e SciELO. Além disso, uma pesquisa manual foi feita nas referências de estudos publicados sobre o assunto. A pesquisa foi realizada em novembro de 2019 com restrições de data da publicação dos últimos dez anos (2009-2019) e também com restrições quanto ao idioma: somente os estudos em português e inglês foram elegíveis. Foram utilizados os termos como: Terapia Manual, Massagem Modeladora, Redução de Medidas, Abdome e Região Abdominal. A estratégia de pesquisa completa usada no PubMed pode ser vista na Tabela 1:

Tabela 1: Estratégia de busca utilizada no PubMed.

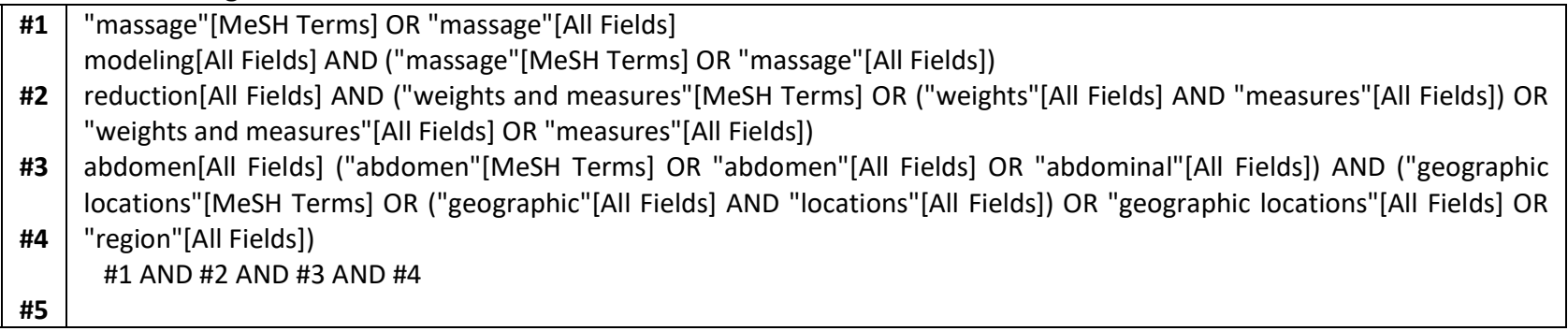

\section{RESULTADOS}

Com base na estratégia de busca, resultou no total de 8244 estudos. Desses 237 restaram para a análise detalhada do artigo, restando sete estudos incluídos que preenchiam todos os critérios de inclusão de elegibilidade previamente estabelecidos. Na Figura 1 é demonstrado o fluxograma dos estudos incluídos 
e a Tabela 1 apresenta as características desses artigos, sendo que estes se apresentam numerados de 1 a 7

e, na discussão, estão incluídos em forma sobrescrita de números ao longo do texto.

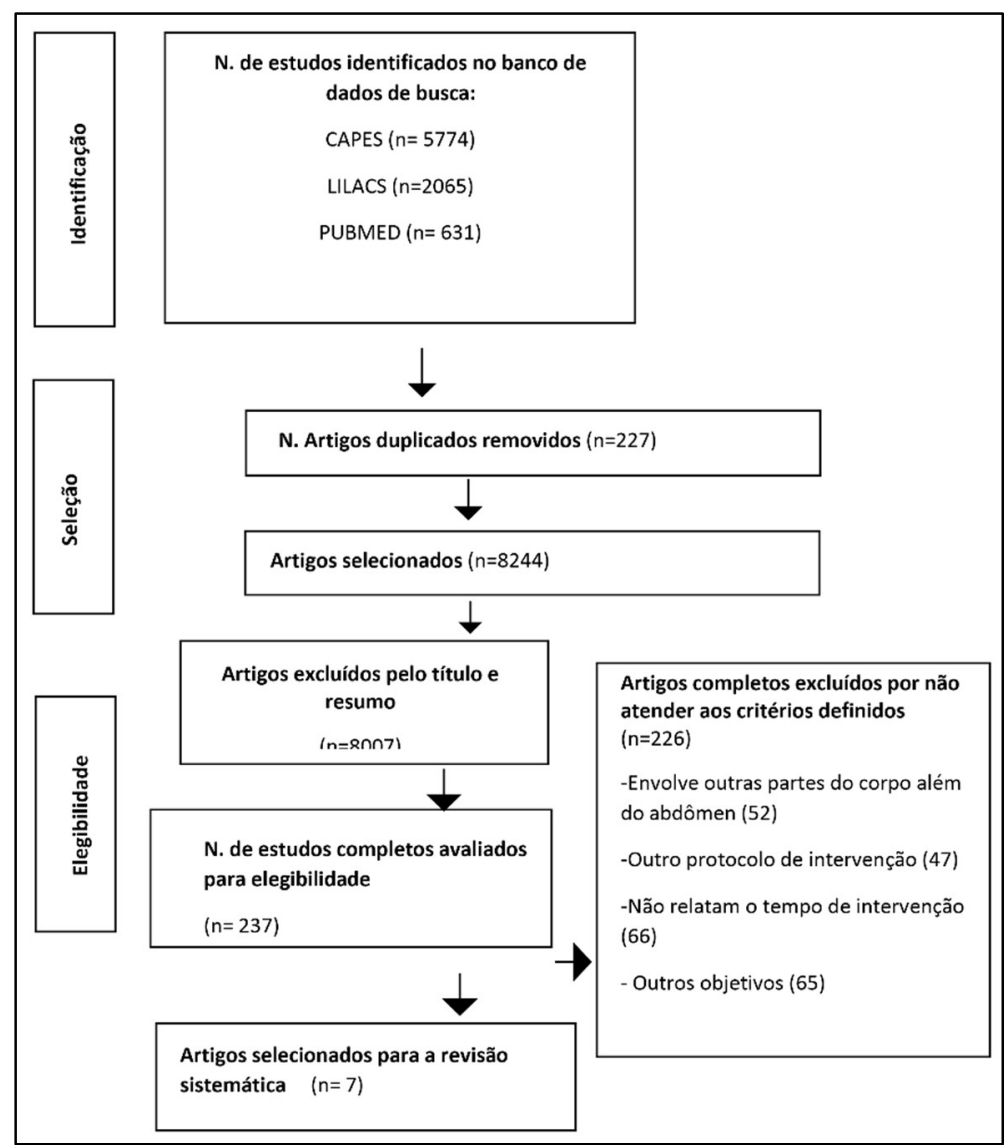

Figura 1: Fluxograma dos estudos selecionados.

Tabela 1: Características dos estudos incluídos nessa revisão.

\begin{tabular}{|c|c|c|c|c|c|c|}
\hline $\mathbf{N}$ & $\begin{array}{c}\text { Autor e data } \\
\text { da } \\
\text { publicação }\end{array}$ & Amostra & Tipo de Estudo & Idade & Protocolo de Intervenção & Conclusão \\
\hline 1 & $\begin{array}{l}\text { TACANI et } \\
\text { al., } 2010\end{array}$ & 10 & Estudo piloto & $\begin{array}{l}20 \text { a } \\
45 \\
\text { anos }\end{array}$ & $\begin{array}{l}\text { Período (Semanas): } 4 ; \\
\text { Número de sessões: 20; } \\
\text { Frequência semanal: 3; } \\
\text { Tempo de sessão: } 20 \text { min; } \\
\text { Tipo de intervenção: } \\
\text { Massagem clássica. }\end{array}$ & $\begin{array}{l}\text { A massagem clássica estética não } \\
\text { produziu redução de massa } \\
\text { corporal IMC, mas contribuiu para } \\
\text { a redução. Houve uma melhora } \\
\text { estatisticamente significativa após } \\
\text { a intervenção no quadril }(p=0,012) \\
\text { e na parede abdominal }(p<0,001) \text {. }\end{array}$ \\
\hline 2 & $\begin{array}{l}\text { MACHADO } \\
\text { et al., } 2017\end{array}$ & 2 & $\begin{array}{l}\text { Estudo } \\
\text { transversal } \\
\text { quantitativo }\end{array}$ & $\begin{array}{l}20 \text { a } \\
40 \\
\text { anos }\end{array}$ & $\begin{array}{l}\text { Período (Semanas): } 8 ; \\
\text { Número de sessões: 16; } \\
\text { Frequência semanal: 2; } \\
\text { Tempo de sessão: } 40 \text { min; } \\
\text { Tipo de intervenção: } \\
\text { Massagem Modeladora. }\end{array}$ & $\begin{array}{l}\text { Os resultados demonstraram } \\
\text { redução na circunferência } \\
\text { abdominal e melhora do aspecto } \\
\text { da pele, a massagem modeladora } \\
\text { é uma técnica eficaz no } \\
\text { tratamento da lipodistrofia } \\
\text { localizada. No estudo não houve } \\
\text { diferença estatisticamente } \\
\text { significativa. }\end{array}$ \\
\hline 3 & $\begin{array}{l}\text { NEVES et al., } \\
2017\end{array}$ & 26 & $\begin{array}{l}\text { Ensaio clínico } \\
\text { randomizado }\end{array}$ & $\begin{array}{l}18 \text { a } \\
30 \\
\text { anos }\end{array}$ & $\begin{array}{l}\text { Período (Semanas): } 2 ; \\
\text { Número de sessões: 10; } \\
\text { Frequência semanal: } 5 ; \\
\text { Tempo de sessão: } 15 \text { min; } \\
\text { Tipo de intervenção: } \\
\text { Massagem modeladora e } \\
\text { Ultrassom. }\end{array}$ & $\begin{array}{l}\text { Os resultados obtidos neste } \\
\text { estudo pode-se afirmar que a } \\
\text { massagem } \\
\text { modeladora é efetiva na redução } \\
\text { da gordura localizada abdominal } \\
\text { nas mulheres. Foi encontrada uma } \\
\text { diferença significativa (superior a } \\
5 \% \text { ). }\end{array}$ \\
\hline 4 & $\begin{array}{l}\text { RAMOS et } \\
\text { al., } 2016\end{array}$ & 2 & $\begin{array}{l}\text { Corte caráter } \\
\text { exploratório }\end{array}$ & $\begin{array}{ll}20 & a \\
45 & \end{array}$ & $\begin{array}{lll}\text { Período } & \text { (Semanas): } & 20 ; \\
\text { Número de sessões: } & 10 ;\end{array}$ & $\begin{array}{l}\text { Os estudos sobre o tema } \\
\text { abordado e os resultados da }\end{array}$ \\
\hline
\end{tabular}




\begin{tabular}{|c|c|c|c|c|c|c|}
\hline & & & & anos & $\begin{array}{l}\text { Frequência semanal:2; } \\
\text { Tempo de sessão: 30min; } \\
\text { Tipo de intervenção: } \\
\text { Massagem modeladora e } \\
\text { Bandagem gessada. }\end{array}$ & $\begin{array}{l}\text { pesquisa evidenciaram a eficácia } \\
\text { do tratamento de gordura } \\
\text { localizada com massagem } \\
\text { modeladora. A diferença não foi } \\
\text { estatisticamente significante. }\end{array}$ \\
\hline 5 & $\begin{array}{l}\text { COSTA et al., } \\
2016\end{array}$ & 30 & $\begin{array}{l}\text { Ensaio clínico } \\
\text { randomizado }\end{array}$ & $\begin{array}{l}18 \text { a } \\
28 \\
\text { anos }\end{array}$ & $\begin{array}{l}\text { Período (Semanas): 16; } \\
\text { Número de sessões: SD; } \\
\text { Frequência semanal: SD; } \\
\text { Tempo de sessão: } 20 \text { min; } \\
\text { Tipo de intervenção: } \\
\text { Massagem modeladora, } \\
\text { endermoterapia } \\
\text { eletrolipólise. }\end{array}$ & $\begin{array}{l}\text { Pode-se concluir que a massagem } \\
\text { modeladora, é realmente efetiva } \\
\text { com a sua aplicação adequada, } \\
\text { por gerar aumento significativo da } \\
\text { temperatura cutânea superficial. } \\
\text { Houve uma diferença significativa } \\
\text { entre as sessões de }(p<0,0001) \text {. }\end{array}$ \\
\hline 6 & $\begin{array}{l}\text { SILVA et al., } \\
2009\end{array}$ & 10 & $\begin{array}{l}\text { Ensaio clínico } \\
\text { Randomizado }\end{array}$ & $\begin{array}{l}20 \text { a } \\
30 \\
\text { anos }\end{array}$ & $\begin{array}{l}\text { Período (Semanas): } 30 ; \\
\text { Número de sessões: 10; } \\
\text { Frequência semanal: 3; } \\
\text { Tempo de sessão: 20min; } \\
\text { Tipo de intervenção: } \\
\text { Massagem modeladora. }\end{array}$ & $\begin{array}{l}\text { A massagem modeladora foi eficaz } \\
\text { na redução perimétrica da } \\
\text { gordura abdominal, sendo } \\
\text { potencializada com ativos. Não } \\
\text { comprovado estatisticamente. }\end{array}$ \\
\hline 7 & $\begin{array}{l}\text { PEREIRA et } \\
\text { al., } 2015\end{array}$ & 11 & $\begin{array}{l}\text { Ensaio clínico } \\
\text { Randomizado }\end{array}$ & $\begin{array}{l}20 \text { a } \\
30 \\
\text { anos }\end{array}$ & $\begin{array}{l}\text { Período (Semanas): SD; } \\
\text { Número de sessões: 10; } \\
\text { Frequência semanal: SD; } \\
\text { Tempo de sessão: 10min; } \\
\text { Tipo de intervenção: } \\
\text { Massagem modeladora e } \\
\text { Ultrassom. }\end{array}$ & $\begin{array}{l}\text { Conclui-se que meio a massagem } \\
\text { modeladora causa melhora visível } \\
\text { do contorno e redução de } \\
\text { medidas, corporal e textura da } \\
\text { pele. A diferença estatisticamente } \\
\text { de significância a } p<0,05 \text {. }\end{array}$ \\
\hline
\end{tabular}

N: Número; SD: Sem Descrição; min: Minutos.

Interpretação e análise dos resultados

Com base na Tabela 1 observa-se que nos últimos dez anos de estudos, não houve um aumento considerável em relação a esta temática, apenas entre os anos de 2016 e 2017 teve mais publicações, não foram publicados mais estudos desde 2017 até o momento. Notando-se que todos os estudos selecionados utilizaram a terapia manual para avaliar a sua efetividade na redução da circunferência abdominal apenas do sexo feminino.

As amostras foram de idades mínimas e máximas encontradas entre as mulheres participantes dos estudos foram de 18 a 45 anos respectivamente e o tamanho amostral dos estudos, compostas de 2 a 30 mulheres. Quanto ao tipo de estudo, 4 deles foram ensaios clínicos randomizados, 1 estudo piloto, 1estudo transversal quantitativo e 1 corte caráter exploratório.

Quanto aos objetivos dos estudos selecionados, quatro deles realizaram comparações de uma terapia com outra, sendo a comparação da terapia manual com o ultrassom, ou duas modalidades de terapia manual, restando apenas três estudos que não realizaram comparações e utilizaram somente uma forma de intervenção.

Os desfechos de dois estudos foi que avaliaram a aplicação da massagem modeladora associada com outro método juntamente com auxílio de cremes, óleos possuindo na sua composição, princípios ativos durante o manuseio da terapia manual sobre a pele das voluntárias, pois os resultados ao final das sessões constataram que a redução da circunferência abdominal era maior que apenas usando a massagem modeladora.

Também dois estudos utilizaram como intervenção a massagem modeladora associada com o ultrassom sendo, que os resultados segundos os estudos são mais relevantes e efetivos, podendo obter maior 
diminuição e uniformização da lipodistrofia localizada. Vale ressaltar que, cinco estudos que compõem esta revisão sistemática relataram que a terapia manual da massagem modeladora, é eficiente na redução da circunferência da região abdominal em mulheres, além de ter melhorado o aspecto da pele no local que foi aplicada a massagem modeladora.

Os estudos $(1,2,3,4,6)$, debateram que devido ao padrão de beleza imposto pela sociedade e pelas próprias mulheres, que dificilmente estão contentes com o seu próprio corpo e suas medidas, ou seja, sempre estão buscando novos tratamentos estéticos. Pois, os benefícios que os tratamentos manuais ou com o uso de aparelho são tentadores oferecendo redução de medida corporal, diminuição da celulite, além de acelerar o metabolismo dentre outras vantagens. As mulheres sempre estão realizando estes tratamentos, como a massagem modeladora e também essas terapias manuais podem até evitar o processo cirúrgico.

\section{DISCUSSÃO}

Realizamos uma revisão sistemática para identificar as evidências científicas acerca da massagem modeladora na remodelagem da circunferência abdominal em mulheres, observamos que os sete estudos incluídos na pesquisa trouxeram esclarece, de que por meio das manobras da massagem modeladora sobre a camada adiposa, chegando até na última camada da pele, resulta na redistribuição das células de gordura, uniformizando o local em que é aplicada a técnica da massagem modeladora, visto que foram utilizadas as técnicas de massagem modeladora em todos os estudos.

Os estudos dessa revisão utilizaram a massagem modeladora como método de intervenção, além que os objetivos foram de verificar os efeitos da massagem modeladora na redução de medidas na região abdominal de mulheres com intervenções de curto prazo e no mínimo de 10 sessões em todos os estudos. Os resultados finais foram satisfatórios entre as voluntárias dos estudos, pois todas diminuíram a sua circunferência abdominal, contorno corporal até mesmo obtiveram um melhor aspecto da pele.

As maneiras de avaliar a circunferência corporal são por meio do adipômetro, fichas de avaliações antes e depois das sessões, pois muitos estudos utilizam estes meios para mensurar a gordura corporal. Para utilizar a fita métrica na região do abdômen são mensurados três locais, sendo demarcados com um lápis dermográfico: supraumbilical (três centímetros acima do umbigo), cicatriz umbilical e infraumbilical (três centímetros abaixo do umbigo). Ainda para o adipômetro, com pinça, mede-se a dobra cutânea sendo uma dobra vertical 2,5 centímetros, mensurada pelos dedos indicadores e polegar calculada a quantidade de gordura subcutânea (GUIRRO et al., 2004). Como no estudo ${ }^{(6)}$ que primeiro fizeram a ficha de avaliação e mediram com a fita métrica, adipômetro e uma câmera digital para um melhor resultado visível.

Nos estudos ${ }^{(1,2,5 \text { e } 6)}$ o tempo de cada sessão foram no mínimo de 20 a 40 minutos que totalizou em 10 sessões, os valores de mensuração no final do tratamento foram de menos cinco centímetros de redução na região abdominal abaixo do umbigo, salientam que nestes estudos foram aplicadas as manobras da massagem modeladora nas participantes, com um profissional que tinha embasamento além de experiência sobre essa técnica para aplicar de forma correta, pois os resultados são mais evidentes (SILVA et al., 2009).

Como nos estudos $(3,4,6)$, utilizaram o tempo de intervenção de 10 sessões com duração de 20 
minutos, e objetivaram avaliar os efeitos da massagem modeladora em mulheres sedentárias que possuem gordura localizada no abdômen, verificando através das sessões a perda de medidas. Em desacordo com estes estudos citados acima, Tacani et al. (2010), concluíram que a massagem não proporcionou resultados satisfatórios como nos demais estudos, pois houve redução de medidas apenas no perímetro do quadril, devido ao tempo de aplicação na região do abdômen ser de apenas 5 min, que provavelmente tenha sido insuficiente comparado com o tempo aplicado no quadril de $14 \mathrm{~min}$ ( 7 min de cada lado).

As idades das amostras variaram em todos os estudos $(1,2,3,4,5,6,7)$, entre 18 a 45 anos, apenas com mulheres, pois são o público que mais busca tratamentos estéticos, e alguns estudos demonstraram que a terapia manual pode beneficiar com resultados durante o decorrer das sessões, sendo associado ao exercício físico ou a alimentação adequada. Alguns estudos ainda investigam a massagem modeladora, devido que se pode obter efeito lipolítico primário ou de forma secundária, mas visto que resulta na melhora da circulação sanguínea, minimiza a ansiedade, dentre outros benefícios desta técnica e associada com exercícios físicos e alimentação os resultados são mais efetivos (TACANI et al., 2010).

Corroborando com os benefícios encontrados da massagem modeladora, Machado et al. (2017), afirmam de ser eficientes nos tratamentos estéticos emagrecedores, pois pode evitar muitas vezes o processo cirúrgico e todos os seus benefícios são abrangentes, como: estimula o metabolismo, aumenta as circulações, elimina as toxinas. Em acordo Tacani et al. (2010), salientam que este tipo de massagem funciona com o sentido centrípeto, de forma constante, mantendo uma pressão e velocidade adequada, além da frequência e tempo para obter os benefícios satisfatórios desta técnica.

Em alguns estudos nos quais as técnicas da massagem modeladora foram associadas a outros métodos como, por exemplo, o uso da aplicação do ultrassom estético $3 \mathrm{MHz}$, no estudo feito em um dos grupos GMUS com sete amostras, obteve uma redução de 7,2 centímetros em relação ao GM de 5,5 centímetros (NEVES et al., 2017). Como no estudo de Pereira et al. (2015), mostraram a eficácia da aplicação da massagem modeladora associada ao ultrassom de $3 \mathrm{MHz}$, onde todas as participantes conseguiram diminuição de medidas da região abdominal de 3 centímetros a mais do que o grupo que recebeu apenas a massagem modeladora.

Para Silva et al. (2009), publicaram que as manobras da massagem modeladora com cremes e óleos contendo princípios ativos proporcionam resultados maiores em perdas de medidas. Concordando com esses estudos, Firmino et al. (2016), acreditam que a massagem modeladora surgiu como um tratamento coadjuvante para a redução de adiposidades proporcionando resultados eficazes e, além disso, aumenta a autoestima de muitas mulheres. Segundo Borges (2006), a massagem modeladora, além de favorecer a remodelação e distribuição de gordura com a utilização de princípios ativos, como cremes e óleos e ativos naturais, oferecem um melhor deslizamento corporal e os benefícios químicos das substâncias utilizadas influenciam decisivamente no resultado da redução de medidas do abdome.

Para Machado et al. (2017), ressaltaram que ao término da intervenção, todas as voluntárias obtiveram redução na circunferência abdominal, além da melhora do aspecto da pele, por isso a técnica foi eficaz no tratamento da lipodistrofia localizada, pois um dos efeitos fisiológicos da massagem é ocasionar o 
aumento de temperatura cutânea, para se ter algum resultado positivo. O que corrobora com os achados de COSTA et al. (2016), observaram que após a cada sessão houve um aumento significativo da temperatura local cutânea superficial, sendo esse um dos efeitos para que as células adiposas se redistribuam em toda a camada, o que concluíram que as voluntárias conseguiram a redução de medidas na região abdominal.

De acordo com Ramos et al. (2016), demonstraram que em seus resultados, evidenciaram que por meio da aplicação da massagem modeladora, sendo com duas voluntárias, onde ambas perderam em torno de menos seis centímetros de medidas do abdômen ao término das sessões, ressaltando a eficácia da massagem modeladora. Em acordo com os mesmos resultados, Valentim et al. (2016), relataram que em seu estudo composto com duas voluntárias, obtiveram os seus resultados semelhantes, na redução da circunferência abdominal cerca de menos seis centímetros ao término da décima sessão, onde constataram a eficácia da massagem e ainda sobre o metabolismo de cada pessoa influência nos resultados podendo ser ainda maior à redução de medidas.

\section{CONCLUSÕES}

De acordo com os resultados obtidos neste estudo, pode-se afirmar que a terapia manual da massagem modeladora é eficaz no tratamento da gordura localizada. Além de todos os seus abrangentes benéficos, tais eles como melhora do fluxo sanguíneo e do linfático, relaxamento do corpo, e eliminação das toxinas do corpo, promove por meio da aplicação das manobras da massagem a remodelação corporal a uniformização da camada adiposa, o que resulta na remodelagem da circunferência corporal, principalmente da região abdominal.

Portanto, sugere-se a realização de novas pesquisas sobre essa temática e que seja de forma mais isolada, com maior número de amostras e maior tempo de tratamento para a validação do estudo. Sendo assim, esta revisão evidencia que o papel do fisioterapeuta na área da dermatofuncional, contribuindo para o enriquecimento e credibilidade dessa terapêutica manual.

\section{REFERÊNCIAS}

BRASIL. Ministério da Saúde. Secretaria de Atenção à Saúde. Departamento de Ações Programáticas Estratégicas. Política nacional de atenção integral à saúde da mulher: princípios e diretrizes. Brasília: Ministério da Saúde, 2018.

BORGES, F. S.. Modalidades terapêuticas nas disfunções estéticas: dermatofuncional. São Paulo: Phorte, 2006.

CASSAR, M.-P.. Manual de Massagem Terapêutica. Rio de Janeiro: Manole, 2001.

COSTA, F. S.. Acupuntura No Tratamento Da Fibromialgia: Revisão da Literatura. Revista Visão Universitária, v.1, n.1, 2017.

COSTA, R.; SCHMILD, D.; VIANA, L. G.; AROCA, G. G. P.; SOUSA, L.. Comparação da termografia cutânea após aplicação da massagem modeladora, endermoterapia e eletrolipólise. ConScientiae Saúde, v.15, n.2, p.241-248, 2016.
FIRMINO, P.; REIS, Y.. A Eficácia da massagem modeladora como uma das técnicas Terapêuticas Usadas no Tratamento do Fibro Edema Geloide. FEPESMIG, 2016.

FRANÇA, I. C.; AKATSUKA, E. W.; LEAL, C. P.; FIGUEIREDO, M. R.; OLIVEIRA, L. I.; ANDRADE, N. S.. Eficácia da Técnica de Massagem Modeladora para Redução De Adiposidades e Fibro Edema Geloide. Atas de Ciências da Saúde, v.4, n.2, 2016.

GONDIM, S. S.; ALMEIDA, M. A. P.. Os Efeitos da Massagem terapêutica manual em pacientes com a síndrome da fibromialgia. Revista Multidisciplinar e de Psicologia, v.12, n.39, 2018

GUIRRO, E. C.; GUIRRO, R.. Fisioterapia Dermato-Funcional. 3 ed. São Paulo: Manole, 2004. 
MACHADO, A. T. O.; NOGUEIRA, A. P. S.; LAÃO, L. T. S.; SANTOS, B. A.; PINHEIRO, L. M.; OLIVEIRA, S. S.. Benefícios da Massagem Modeladora na Lipodistrofia Localizada. Id on Line Rev. Psic., v.11, n.35, 2017.

MILANI, G. B.; JOÃO, S. M. A.; FARAH, E. A.. Fundamentos da fisioterapia dermato-funcional: revisão de literatura. Revista Fisioterapia e Pesquisa, São Paulo, v.13, n.1, p.37-43, 2006.

NEVES, S. R.; OLIVEIRA, D.. Eficácia da associação de técnicas manuais e eletrotermoterapia na redução de medidas do abdômen. Revista de Biologia e Saúde da UNISEP, v.1, n.1, p.67-71, 2007.

PEREIRA, P.; CÉZAR, J. L. F.; OLIVEIRA, H. S.; DELFINO, M. M.. Endermoterapia e Ultrassom Terapêutico associado à massagem modeladora na redução de medidas abdominais. Revista da Universidade Vale do Rio Verde, v.13, n.2, 2015. DOI: http://dx.doi.org/10.5892/ruvrd.v13i1.2260

PEREIRA, F.. Eletroterapia sem mistérios: aplicações em estética facial e corporal. 3 ed. Rio de Janeiro: Rubio, 2007.

RAMOS, D.; FERNANDES, E. M. S.; OLIVEIRA, M. I. R.; OLIVEIRA, G. L.; FRANCO, M. O.. Redução de medidas com auxílio da massagem modeladora associada a ativos lipolíticos e bandagem gessada. In: ENCONTRO LATINO AMERICANO DE INICIAÇÃO CIENTÍFICA, 13; ENCONTRO LATINO AMERICANO DE PÓS-GRADUAÇÃO, 9. Anais. São José dos Campos: Universidade do Vale do Paraíba, 2016. p.1-5.
STARKEY, C.. Recursos Terapêuticos em Fisioterapia. 2 ed. São Paulo: Manole, 2001.

SILVA, T. C.; SILVA, Y. F. O.. A Dermato-Funcional no ensino de graduação em fisioterapia: visão de profissionais atuantes na cidade de Inhumas/GO. In: SEMINÁRIO SOBRE DOCÊNCIA UNIVERSITÁRIAM, 1. Anais. Anápolis: Universidade Estadual de Goiás, 2011.

SILVA, R. M. V.; MEYER, P. F.; SOUZA, J. F.; FÉLIZ, J. L. O.; DELGADO, E. B. S.; QUEIROZ, L. O.; MEDEIROS, M. L.. Efeitos da fonoforese com gel de ativos lipolíticos na adiposidade abdominal. São Paulo, 2014.

TACANI, P. M.; MACHADO, A. F. P.; SOUZA, D. A. A.; TACANI, R. E.. Efeito da massagem clássica estética em adiposidades localizadas: estudo piloto. Fisioterapia e Pesquisa, São Paulo, v.17, n.4, p.352-7, 2010.

TRIFFONI, A. M.; MONTE-ALEGRE, F. A.; MERHI, V. A. L.; GARCIA, R. W. D.. Additional abdominal measurements are a useful tool to evaluate body composition in obese women. Arq. Gastroenterol., São Paulo, v.56, n.3, 2019. DOI: http://dx.doi.org/10.1590/s0004-2803.201900000-5

VALENTIM, C.; NASCIMENTO, D.; VASCONCELOS, L.; ANDRADE, D.; EMELLY, R.. Eficácia da atadura gessada associada à massagem modeladora para tratamento de gordura localizada. Fisioterapia e Terapia Ocupacional, 2016.

A CBPC - Companhia Brasileira de Produção Científica (CNPJ: 11.221.422/0001-03) detém os direitos materiais desta publicação. Os direitos referem-se à publicação do trabalho em qualquer parte do mundo, incluindo os direitos às renovações, expansões e disseminações da contribuição, bem como outros direitos subsidiários. Todos os trabalhos publicados eletronicamente poderão posteriormente ser publicados em coletâneas impressas sob coordenação da Sustenere Publishing, da Companhia Brasileira de Produção Científica e seus parceiros autorizados. Os (as) autores (as) preservam os direitos autorais, mas não têm permissão para a publicação da contribuição em outro meio, impresso ou digital, em português ou em tradução. 